\title{
Características clínicas, endoscópicas, manométricas y magnitud de reflujo gastroesofágico ácido patológico en pacientes con esófago en cascanueces. Análisis prospectivo de 80 pacientes
}

\author{
Attila Csendes, Carlos Cárcamo, Ana Henríqueza. \\ Nutcracker esophagus. \\ Analysis of 80 patients
}

Background: The nutcracker esophagus, a primary motor disorder, is frequently associated with noncardic chest pain. Aim: To study the clinical, endoscopic, manometric, and pathological features and $24 \mathrm{~h}$ acid reflux in patients with nutcracker esophagus. Patients and methods: Eighty patients (63 females, aged 26 to 70 years) with nutcracker esophagus, defined as the presence of contraction waves of more than $180 \mathrm{mmHg}$ in the esophageal manometry, were studied. All were subjected to an upper gastrointestinal endoscopy, $24 \mathrm{~h}$ esophageal $\mathrm{pH}$ monitoring and a new manometric study. Results: Eighty percent of patients had symptoms suggestive of gastroesophageal reflux, 31\% had retrosternal pain and $25 \%$ had dysphagia. Upper endoscopy was normal in $41 \%$ and showed erosive esophagitis or Barrett esophagus in 27\%. Fifty percent of patients had an abnormal acid reflux. There were no significant differences in manometric parameters between patients with and without acid reflux. Conclusions: Symptoms of gastroesophageal reflux not necessarily mean that a pathological reflux is present. Primary motor disorders of the esophagus may cause similar symptoms (Rev Méd Chile 2004; 132: 160-4).

(Key Words: Esophageal motility disorders; Esophagitis; Manometry).

Recibido el 16 de mayo, 2003. Aceptado en versión corregida el 23 de octubre, 2003.

Departamento de Cirugía, Hospital Clínico Universidad de Chile. Santiago, Chile.

aTecnólogo Médico

$\mathrm{E}^{\mathrm{n}}$ 1977, Brand y cols ${ }^{1}$ describieron en un grupo de pacientes con dolor torácico de tipo anginoso y coronariografía normal, alteraciones manométricas del esófago. Posteriormente Benja-

Correspondencia a: Dr. Attila Csendes, Facs. Departamento de Cirugía, Hospital Clínico Universidad de Chile. Santos Dumont \#999, Santiago, Chile. Fono: 56-2-7774387. Fax: 56-2-7775043. E mail: acsendes@machi.med.uchile.cl min y cols ${ }^{2}$ confirmaron esta anomalía motora, denominándolo esófago en cascanueces, término que se emplea en la actualidad. Constituye el hallazgo manométrico anormal más común en pacientes referidos a estudio manométrico por dolor torácico no cardíaco ${ }^{3}$. El diagnóstico de esta afección se basa exclusivamente en los hallazgos manométricos, por este motivo, es tardío y probablemente realizado en una minoría de pacientes 
en nuestro medio, ya que los laboratorios adecuadamente equipados de manometría computarizada de esófago son muy pocos en Chile.

El objetivo del presente estudio prospectivo fue determinar las características clínicas, los diagnósticos de derivación hacia el estudio manométrico, los hallazgos endoscópicos y principalmente evaluar la presencia de reflujo ácido patológico hacia el esófago distal, en un grupo de pacientes con esófago en cascanueces.

\section{MATERIAL Y MÉTODO}

Pacientes. En un período comprendido entre julio de 1999 y julio de 2002 se realizaron 543 estudios manométricos de una manera estándar, registrando las características clínicas y los diagnósticos de derivación para efectuar el estudio manométrico. De estos estudios, 80 pacientes $(14,7 \%)$ demostraron tener las características de un esófago en cascanueces y constituyeron la base del presente estudio. Todos ellos aceptaron ser incluidos en el presente estudio, realizándose una endoscopia digestiva alta y un estudio de reflujo ácido durante las $24 \mathrm{~h}$.

Endoscopia. Este procedimiento se realizó en todos los pacientes después de una anestesia tópica faríngea y premedicación con midazolam y Buscapina ${ }^{\circledR}$. Se determinó las características de la mucosa esofágica, gástrica y duodenal, excluyendo a pacientes con patología péptica, gástrica 0 duodenal. Los hallazgos endoscópicos se catalogaron en normales, esofagitis erosiva (presencia de 1 o más erosiones aisladas en esófago distal) epitelio columnar corto en esófago distal (presencia de mucosa columnar tapizando 3 o menos $\mathrm{cm}$ del esófago distal) y hernia hiatal (presencia de un saco gástrico herniado en esófago distal).

Análisis manométricos. Este procedimiento fue realizado en todos los pacientes después de $12 \mathrm{~h}$ de ayuno y con leve anestesia faríngea. El sensor manométrico consta de 4 catéteres de polivinilo con orificios laterales de $2 \mathrm{~mm}$ separados a $5 \mathrm{~cm}$ de distancia (Synectics Medtronic, Estocolmo, Suecia), perfundidos a un flujo de $0,2 \mathrm{ml} / \mathrm{min}$ desde una bomba pneumo-hidraúlica. Estos catéteres van conectados a un polígrafo conectado a un sistema computarizado que analiza todas las características motoras del esófago. Se determinó la presión de reposo del esfínter esofágico inferior (en $\mathrm{mmHg}$ ), el largo total y el largo abdominal (en $\mathrm{mm})$, la amplitud de las ondas de contracción de la mitad distal y de la mitad proximal del esófago (en $\mathrm{mmHg}$ ), la duración (en segundos) y el porcentaje de ondas de contracción peristálticas después de la deglución de $5 \mathrm{ml}$ de agua tibia. Los resultados de todos los registros son analizados mediante el programa computacional. Estos estudios fueron realizados por una tecnóloga médica con más de 25 años de experiencia en manometría esofágica. El esófago en cascanueces se definió por la presencia de una o más ondas de contracción con amplitud mayor a $180 \mathrm{mmHg}^{4,5}$.

$\mathrm{PH}$ de 24 h. Con el objeto de determinar la relación entre reflujo ácido patológico y el trastomo motor esofágico, en todos los pacientes se realizó el estudio de reflujo ácido durante $24 \mathrm{~h}$, inmediatamente después del estudio manométrico, una vez precisada la ubicación del esfínter esofágico inferior. El electrodo de antimonio (Synectics Metronic, Estocolmo, Suecia) se colocó $5 \mathrm{~cm}$ por encima del límite proximal del esfínter, conectado a un sistema de registro continuo durante $24 \mathrm{~h}$ (Digitrapper II). El paciente recibía instrucciones precisas con respecto a su hábito de sueño, comida y presencia de síntomas. Los parámetros que se analizaron fueron los siguientes ${ }^{6}$ :

a. Porcentaje del tiempo con $\mathrm{pH}<4$ en posición de pie (normal 1,9\%).

b. Porcentaje del tiempo con $\mathrm{pH}<4$ en posición supina (normal 1,2\%).

c. Porcentaje del tiempo con $\mathrm{pH}<4$ durante comidas (normal 3\%).

d. Porcentaje del tiempo con $\mathrm{pH}<4$ postprandial (normal 2,1\%).

e. Porcentaje total del tiempo con $\mathrm{pH}<4$ en $24 \mathrm{~h}$ (normal $<4 \%$ ).

f. Score de DeMeester (normal <4\%).

De todos estos parámetros los más útiles $\mathrm{y}$ prácticos son el porcentaje de tiempo total en $\mathrm{pH}$ $<4$ de $24 \mathrm{~h}$ y el score de DeMeester. Los resultados se clasificaron como ausencia de reflujo ácido patológico (4\% ó menos del tiempo con $\mathrm{pH}<4$ en $24 \mathrm{~h}$, y que corresponde a $55 \mathrm{~min}$ ) o presencia de reflujo ácido patológico (más de $4 \%$ del tiempo con $\mathrm{pH}<4$ en $24 \mathrm{~h}$ ). 
Análisis estadístico: Para los cálculos de significación estadística, se empleó el test exacto de Fisher y el Chi cuadrado, tomando un $p<0,05$ como significativo.

\section{RESULTADOS}

En la Tabla 1 se señalan los diagnósticos con los cuales se enviaron estos 80 pacientes para estudio manométrico. El 43,7\% presentaba un síndrome clínico de reflujo gastroesofágico (RGE), mientras que $56,3 \%$ fue enviado al estudio manométrico por la presencia de dolor torácico $(31,3 \%)$ o por disfagia (25\%). Llama la atención que 12,5\% de los pacientes había sido operado por RGE (sin estudio manométrico previo) y persistía con dolor torácico. Sólo 3 pacientes habían sido estudiados con coronariografía previa normal y señalados como dolor torácico en estudio. El resto de los pacientes con dolor torácico no coronario (22 casos) había sido evaluado previamente por cardiólogo. Las características clínicas de todos los pacientes incluidos en el presente estudio se muestran en la Tabla 2. Los síntomas de RGE (pirosis o regurgitación) estuvieron presentes en un gran porcentaje de los pacientes. Dolor retroesternal y disfagia se presentaron en más de $1 /$ 4 de los casos. Pacientes con diagnóstico agregado de síndrome de intestino irritable correspondieron casi a 89\%, así como el compromiso psicológico de ansiedad, presente en $61 \%$ de los casos. De este grupo, 44 pacientes (53\%) habían estado previamente en tratamiento psiquiátrico.

Los hallazgos endoscópicos se observan en la Tabla 3. Endoscopia normal se detectó en $41 \%$ de los pacientes, mientras que una esofagitis erosiva o un epitelio columnar corto tapizando el esófago distal se presentaron en un porcentaje similar.

En la Tabla 4 se muestran los resultados del estudio manométrico en pacientes con y sin reflujo patológico. En el presente estudio 40 pacientes (50\%) presentaron reflujo ácido patológico con un valor promedio de $11,8 \%$ del tiempo con $\mathrm{pH}<4$ en 24, con un score de DeMeester de 41,9. El 50\% restante demostró un reflujo ácido dentro de límites normales. No hubo ninguna diferencia significativa en ninguno de los parámetros manométricos analizados.
Tabla 1. D iagnóstico de derivación para el estudio manométrico. $\mathrm{N}=80$

\begin{tabular}{|lll|}
\hline & N & $\%$ \\
\hline Síntomas de RGE & 35 & 43,7 \\
Síntomas de RGE y disfagia & 20 & 25 \\
Trastornos motores & & \\
$\quad$ esófago con dolor torácico & 15 & 18,75 \\
RGE operado con dolor torácico & 10 & 12,5 \\
\hline
\end{tabular}

RGE: reflujo gastroesofágico

Tabla 2. C aracterísticas clínicas. $\mathbf{N}=80$

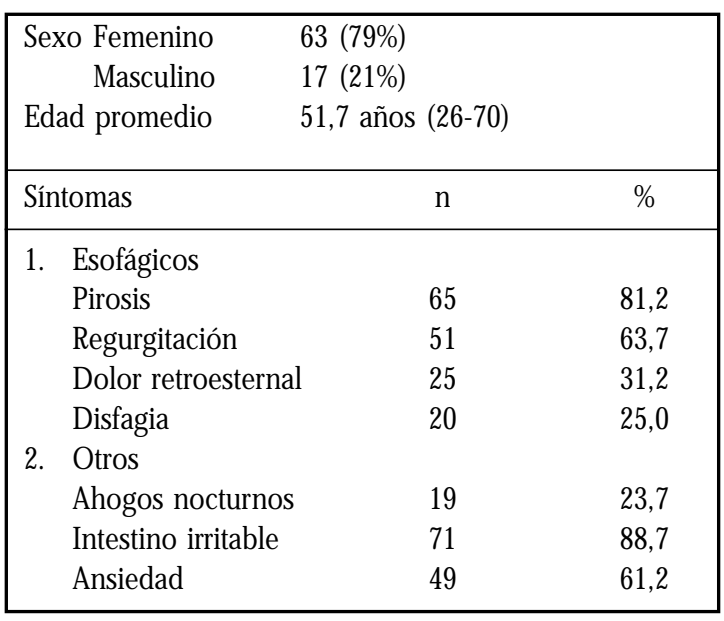

Tabla 3. $\mathrm{H}$ allazgos endoscópicos y biópticos. $\mathbf{N}=80$

\begin{tabular}{|c|c|c|}
\hline Endoscopia & $\mathrm{n}$ & $\%$ \\
\hline 1. Endoscopia normal & 33 & 41,2 \\
\hline 2. Esofagitis erosiva & 21 & 26,2 \\
\hline 3. Epitelio columnar corto en esófago distal & 22 & 27,5 \\
\hline 4. Hemia hiatal & 4 & 5,0 \\
\hline
\end{tabular}

\section{DisCUSIÓN}

El esófago en cascanueces es el trastomo motor primario más frecuente en pacientes que son enviados a un laboratorio de manometría esofágica por dolor torácico no cardíaco ${ }^{1-3,7,8}$. Desconocemos la prevalencia del esófago en cascanueces en sujetos normales, debido a que no hay estudios referentes a este punto. Probablemente se debe al hecho que al encontrar una manometría compatible con un esófa- 
Tabla 4. C aracterísticas manométricas

\begin{tabular}{|llll|}
\hline & Sin reflujo GE (N=40) & Con reflujo GE (N=40) & $\mathrm{p}$ \\
\hline Pr. EGE (mmHg) & $13,4 \pm 4,9$ & $14,5 \pm 7,2$ & $\mathrm{~ns}$ \\
Largo total (mm) & $39 \pm 0,8$ & $37 \pm 0,9$ & $\mathrm{~ns}$ \\
Largo abdominal (mm) & $9 \pm 0,4$ & $6 \pm 0,5$ & $\mathrm{~ns}$ \\
Amplitud ondas distales (mmHg) & $138,3 \pm 26,5$ & $137 \pm 31,2$ & $\mathrm{~ns}$ \\
Duración (seg) & $5,6 \pm 1,2$ & $5,2 \pm 1,3$ & $\mathrm{~ns}$ \\
\% peristalsis & 94 & 94 & $\mathrm{~ns}$ \\
Amplitud ondas proximales (mmHg) & $73,1 \pm 17,7$ & $77,6 \pm 19,9$ & $\mathrm{~ns}$ \\
Duración (seg.) & $5,6 \pm 0,7$ & $2,5 \pm 0,8$ & $\mathrm{~ns}$ \\
\% peristalsis & 98 & 98 & $\mathrm{~ns}$ \\
Pr. esfínter cricofaríngeo (mmHg) & $97,3 \pm 32,0$ & $107 \pm 33,7$ \\
Amplitud ondas faríngeas (mmHg) & $62,0 \pm 21,3$ & $64 \pm 22,0$ & \\
\hline
\end{tabular}

ns $=$ no significativo. EGE $=$ Esfínter gastroesofágico .

go en cascanueces, no se considera dentro de un grupo control o normal. En un estudio publicado hace 10 años, considerando 109 controles, en ninguno existió la presencia de esófago en cascanueces $^{9}$. El mecanismo fisiopatológico que causa este trastorno motor es desconocido ${ }^{10}$ así como el origen del dolor torácico asociado a esta condición. La mayoría de los autores consideran que el segmento más comprometido del esófago torácico es la porción distal ${ }^{11,12}$, aun cuando puede haber compromiso aislado del segmento proximal del esófago torácico ${ }^{11}$. Estudios recientes han señalado que el diagnóstico manométrico se basa en la presencia de 1 o más ondas de contracción peristálticas mayores de $180 \mathrm{mmHg}^{12-15}$, que fue el criterio empleado en el presente estudio.

Las características clínicas en estudios actuales ${ }^{12-15}$ son muy similares a los resultados del nuestro: la mayoría son mujeres y en una edad promedio que se reproduce en todos las publicaciones, entre 50 y 55 años. Los síntomas por lo que consultan pueden corresponder a dolor torácico 0 disfagia $^{12,14}$ o por reflujo gastroesofágico ${ }^{13}$ como ocurrió con alta frecuencia en nuestro material. El estudio endoscópico, en la mayoría de los casos es normal y esofagitis erosiva se describe en $5 \%{ }^{13} u$ $8 \% 14$. Nosotros tuvimos un porcentaje más alto de esofagitis erosiva, probablemente asociado a la presencia de un reflujo ácido patológico.

Hay varios autores que destacan la relación del esófago en cascanueces con trastornos psicológicos o factores relacionados con estrés ${ }^{16,18}$, hecho que estuvo presente muy frecuentemente en nuestros pacientes. El diagnóstico asociado de síndrome de intestino irritable se presentó en 89\% de los pacientes, como se ha descrito previamente ${ }^{17,18}$. Por eso, se ha sugerido el empleo de bloqueadores de los canales de calcio con el propósito de disminuir la amplitud de las contracciones esofágicas y aliviar el dolor, pero con resultados muy inconsistentes y disímiles ${ }^{19}$. Por estas discrepancias y resultados desalentadores, hay autores que han postulado que el esófago en cascanueces puede ser una enfermedad relacionada con un reflujo ácido patológico más que una alteración motora primaria. Así Achem y cols ${ }^{11}$ encontraron $35 \%$ de sus pacientes con reflujo patológico, con una respuesta subjetiva adecuada en $80 \%$ a los medicamentos ácido-supresores. Más recientemente, Borgesson y cols ${ }^{13}$ se plantearon los mismos conceptos, basados en el hecho que 49\% de 43 pacientes presentaron un reflujo ácido patológico. En nuestro estudio, los síntomas de RGE fueron frecuentes en el grupo de 80 pacientes, con 40 de ellos (50\%) presentando reflujo ácido patológico hacia el esófago distal. Silva y cols $^{14}$ también describen $41 \%$ de $\mathrm{pH}$ de $24 \mathrm{~h}$ anormal en un grupo de 97 pacientes con esófago en cascanueces. Sin embargo, vale la pena recalcar, nuevamente, que los síntomas sugerentes de RGE (pirosis o regurgitación) no necesariamente significan la existencia de un reflujo patológico, ya que la pirosis estuvo presente en $81 \%$ de los pacientes, pero sólo la mitad presentó un reflujo patológico. Por este motivo destacamos muy fuertemente la necesidad de realizar un estudio 
manométrico y pH de $24 \mathrm{~h}$ en aquellos pacientes que se consideran candidatos a cirugía antirreflujo, ya que los síntomas pueden corresponder a trastornos motores y no necesariamente a un reflujo patológico. De hecho, en nuestro material, $12 \%$ había sido operado con cirugía antirreflujo sin estudios funcionales previamente, con persistencia de pirosis y dolor retroesternal.

El mecanismo que induce esta anormalidad motora en respuesta a un reflujo ácido patológico se desconoce. Se postula que normalmente el material refluido hacia el esófago es limpiado por la

\section{REFERENCIAS}

1. BRAND DL, MARTIN D, Pope CEII. Esophageal manometrics in patients with angina-like chest pain. Am J Dig Dis 1977; 22: 300-4.

2. Benjamin SB, Gerhardt DC, CASTell DO. High amplitude peristaltic esophageal contractions associated with chest pain and/or dysphagia. Gastroenterology 1979; 77: 478-83.

3. FANY J, BYoKMAN D. Nutcracker esophagus: GERD or an esophageal motility disorder. Am J Gastroent 2002; 97: 1556-7.

4. Katz PO, Castell JA. Nonachalasic motor disonders. En: Castell DO; Richter J. Editors. The esophagus, $3^{\text {rd }}$ Edition, Philadelphia, Lippincott, Williams y Wilkins Ed. 1999; 215-34.

5. CASTEL JA. Esophageal manometry. In: Castell DO Editor, The esophagus, $2^{\text {nd }}$ Edition, Boston, Little Known Co. Editors 1995; 133-52.

6. Alvarez F, Burdiles P, Braghetto I, Henríouez A, Quesada S, CSENDES P. Magnitud del reflujo gastroesofágico a ácido cuantificado por medición de $\mathrm{pH}$ de $24 \mathrm{~h}$ según el grado de esofagitis endoscópica. Rev Méd Chile 1994; 122: 59-67.

7. Traube M, Aibibi R, Mc Camum RW. High amplitude peristaltic esophageal contractions associated with chest pain. JAMA 1983; 250: 2655-9.

8. Katz PO, Dalton CB, Richter JE, Win WC, Castell DO. Esophageal testing of patients with noncardiac chest pain or dysphagia. Results of three years expenence with 1161 patients. Ann Intern Med 1987; 106: 593-7.

9. Csendes A, Maluenda F, Braghetto I, Csendes P, Henríquez A, Quesada S. Location of the lower oesophageal sphincter and the squamous columnar mucosal junction in 109 healthy controls and 778 patients with different degrees of endoscopic esophagitis. Gut 1993; 34: 21-7. actividad peristáltica primaria y secundaria. Se piensa que el esófago en cascanueces puede ser una respuesta exagerada de este mecanismo normal, gatillado o estimulado por factores tensionales y por una sensibilidad visceral aumentada ${ }^{20}$. Nosotros estamos en estos momentos realizando un seguimiento de pacientes con esófago en cascanueces y reflujo ácido patológico, tratándoles con inhibidores de la bomba de protones, con el objeto de comprobar si hay mejoría o desaparición de la sintomatología y del trastorno motor 1 a 2 años después de un tratamiento médico continuo.

10. KAHRIIAS PJ. Esophageal motility disorders. Cument concepts of pathogenesis and treatment. Can J Gastroent 2000; 14: 221-31.

11. Achem SR, Kolts BE, BuRTon L Segmental versus diffuse nutcracker esophagus. An intermitent motility pattern. Am J Gastroent 1993; 88: 847-51.

12. Pilhall M, Borgesson M, Rolng P, Mannheimer C. Diagnosis of nutcracker esophagus, segmental or diffuse hypertensive patterns and clinical presentation. Dig Dis Sci 2002; 47: 1381-8.

13. Borgesson M, Pilhall M, Roung P, Mannheimer C. Gastroesophageal acid reflux in patients with nutcracker esophagus. Scand J Gastroent 2001; 36: 916-20.

14. Sileva LF, LEMmon EM. Esófago en gueba-nozes. Am Q G A 2000; 37: 217-23.

15. Bassatti G, Fiorelia S, German U, Rosem P, Battagla E, Morew A. The nutcracker esophagus: a late diagnostic yield not with standing chest pain and dysphagia. Dysphagia 1998; 13: 213-7.

16. Clouse RE, Lustman PJ. Psychiatric illness and contractions abnormalities of the esophagus. N Eng J Med 1983; 309: 42.

17. Richter JE, Obrecht WF, Bradiey LA, Young LD, ANDERSON KO. Psychological comparison of patients with nutcracker esophagus and imitable bowel syndrome. Dig Dis Sci 1986; 31: 131-8.

18. Anderson KO, Dalton CB, Bradiey LA, Richter JE. Stress induces alterations of esophageal pressures in healthy volunteers and non cardiac chest pain patients. Dig Dis Sci 1989; 34: 83-91.

19. Cattan EL, Castell DO, Johnson DA, Spinlng TJ, Hirszel R, ChaBARIAI JJ. Diltiazem therapy for symptoms associated with nutcracker esophagus. Am J Gastroent 1991; 86: 272-6.

20. Richter JE, Barish CE, Castell DO. Abnormal sensory perception in patients with esophageal chest pain. Gastroenterology 1986; 91: 845-52. 